J Chem Theory Comput. 2016 November 08; 12(11): 5411-5421. doi:10.1021/acs.jctc.6b00552.

\title{
All-atom Continuous Constant pH Molecular Dynamics With Particle Mesh Ewald and Titratable Water
}

\author{
Yandong Huang ${ }^{\dagger}$, Wei Chen ${ }^{\dagger}$, Jason A. Wallace ${ }^{\ddagger}$, and Jana Shen ${ }^{\dagger}$ \\ tDepartment of Pharmaceutical Sciences, University of Maryland School of Pharmacy, Baltimore, \\ $\mathrm{MD}$ \\ ‡University of Oklahoma College of Dentistry, Oklahoma City, OK
}

\begin{abstract}
Development of a $\mathrm{pH}$ stat to properly control solution $\mathrm{pH}$ in biomolecular simulations has been a long-standing goal in the community. Towards this goal recent years have witnessed the emergence of the so-called constant $\mathrm{pH}$ molecular dynamics methods. However, the accuracy and generality of these methods have been hampered by the use of implicit-solvent models or truncation-based electrostatic schemes. Here we report the implementation of the particle mesh Ewald (PME) scheme into the all-atom continuous constant $\mathrm{pH}$ molecular dynamics (CpHMD) method, enabling CpHMD to be performed with a standard MD engine at a fractional added computational cost. We demonstrate the performance using $\mathrm{pH}$ replica-exchange $\mathrm{CpHMD}$ simulations with titratable water for a stringent test set of proteins, HP36, BBL, HEWL and SNase. With the sampling time of $10 \mathrm{~ns}$ per replica, most $\mathrm{p} K_{\mathrm{a}}$ 's are converged, yielding the average absolute and root-mean-square deviations of 0.61 and 0.77 , respectively, from experiment. Linear regression of the calculated vs experimental $\mathrm{p} K_{\mathrm{a}}$ shifts gives a correlation coefficient of 0.79 , a slope of 1 and an intercept near 0 . Analysis reveals inadequate sampling of structure relaxation accompanying a protonation-state switch as a major source of the remaining errors, which are reduced as simulation prolongs. These data suggest PME-based CpHMD can be used as a general tool for $\mathrm{pH}$-controlled simulations of macromolecular systems in various environments, enabling atomic insights into $\mathrm{pH}$-dependent phenomena involving not only soluble proteins but also transmembrane proteins, nucleic acids, surfactants and polysaccharides.
\end{abstract}

\section{Graphical abstract}

Correspondence to: Jana Shen.

Supporting Information Available

Additional analysis (tables and figures). This material is available free of charge via the Internet at http://pubs.acs.org. This material is available free of charge via the Internet at http://pubs.acs.org/. 


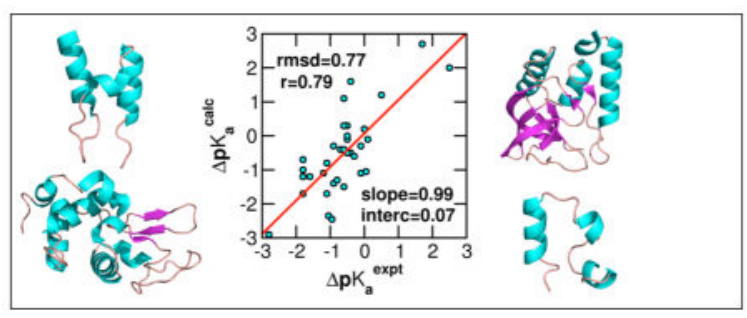

\section{Introduction}

Extending thermodynamic ensembles to include constant solution $\mathrm{pH}$ has been a longstanding goal in the advancement of molecular dynamics (MD) methodologies. Towards this goal two constant $\mathrm{pH}$ frameworks have been developed over the past decade to offer control of solution $\mathrm{pH}$ while propagating atomic positions. The first framework, referred to as discrete constant $\mathrm{pH} \mathrm{MD}$, combines MD with Monte-Carlo sampling of discrete protonation states, ${ }^{1-4}$ while the second one, referred to as continuous constant $\mathrm{pH}$ MD (CpHMD), makes use of the $\lambda$-dynamics ${ }^{5}$ based extended Hamiltonian to propagate a set of continuous titration coordinates. ${ }^{6,7}$ Until recently, all constant $\mathrm{pH}$ methods relied on dielectric continuum models such as Poisson-Boltzmann theory ${ }^{1}$ or generalized-Born implicit-solvent models for sampling protonation states, while either continuum models ${ }^{2,6,7}$ or explicit-solvent description was used for conformational dynamics. ${ }^{1,3,8}$ The latter is also referred to as the hybrid-solvent constant $\mathrm{pH}$ MD. These methods enabled $\mathrm{p} K_{\mathrm{a}}$ predictions of proteins ${ }^{9,10}$ as well as lipids and surfactants, ${ }^{11,12}$ and mechanistic studies of pH-coupled conformational dynamics (see a recent review ${ }^{13}$ for references). However, the underlying approximations in the implicit-solvent models hinders the systematic improvement of accuracy and generalization to systems that necessitate fully explicit-solvent description, for example, highly charged molecules such as nucleic acids and those involving heterogeneous dielectric environments such as transmembrane proteins.

To overcome the limitations imposed by continuum models, the CpHMD framework has been recently extended to fully explicit-solvent (all-atom) simulations by three groups. ${ }^{14-16}$ In the work of Donnini et al, which was implemented in the GROMACS program, ${ }^{17}$ the particle mesh Ewald (PME) scheme ${ }^{18,19}$ was used to model long-range electrostatics in conformational dynamics; however, it was not mentioned as to whether PME forces were applied to $\lambda$ dynamics. ${ }^{14}$ The development was tested on several model compounds with a single titratable site. ${ }^{14}$ Making use of the force shifting scheme for electrostatics in both conformational and $\lambda$ dynamics, CpHMD based on multi-site $\lambda$ dynamics, ${ }^{15}$ which was implemented in CHARMM,${ }^{20}$ has been developed and validated using $\mathrm{p} K_{\mathrm{a}}$ calculations of a ribozyme ${ }^{21}$ proteins ${ }^{22}$ and a transmembrane helix. ${ }^{23}$ The development of Shen and coworkers, ${ }^{16}$ which was also implemented in CHARMM,${ }^{20}$ utilized the generalized reaction field (GRF) scheme by Tironi et al. ${ }^{24}$ for both conformational and $\lambda$ dynamics, and it was validated based on $\mathrm{p} K_{\mathrm{a}}$ calculations of proteins, ${ }^{25}$ surfactants embedded in micelle and bilayer environments, ${ }^{26}$ and sol-gel transition of polysaccharides. ${ }^{27}$ Another major difference between the latter and former two groups' work is with respect to the system net charge. While the latter utilized co-titrating ions ${ }^{16}$ and later titratable water ${ }^{25}$ to ensure 
charge neutrality, the other two developments neglected the effect of fluctuating net charge in the system.

The objective of this work is to develop the PME-based all-atom CpHMD and validate the implementation using $\mathrm{p} K_{\mathrm{a}}$ calculation of a set of proteins including a challenging one, staphylococcal nuclease (SNase), which has not been demonstrated using force shifting ${ }^{15}$ or GRF-based CpHMD. ${ }^{25}$ Our motivation is several fold. First, GRF schemes invoke a spherical representation, ${ }^{24,28}$ which is not suitable for anisotropic systems such as those involving membranes which are a major application target of constant $\mathrm{pH}$ MD. In fact, the use of GRF in membrane protein simulations (with CHARMM27 lipid force field ${ }^{29}$ ) resulted in significant shrinkage of the bilayer in terms of area per lipid (unpublished data), although lipid bilayers are known to be very sensitive to simulation setting and CHARMM27 was developed using PME. ${ }^{29}$ Second, our previous analysis showed force shifting or GRF-based CpHMD favors neutral species in titration due to the underestimation of favorable charging free energies and solvation free energies of charged solutes. ${ }^{30}$ This may be a major cause for the systematic overestimation of $\mathrm{p} K_{\mathrm{a}}$ shifts of buried residues, ${ }^{25}$ although we acknowledge that the deviations may be reduced by performing the finite-size correction for charging free energies as shown by Reif et al. ${ }^{31,32}$ in the context of simulations with the Baker-Watts reaction field. ${ }^{28}$ Lastly, although it remains somewhat debatable as to whether PME outperforms GRF for charged proteins, ${ }^{33,34}$ PME is arguably the most widely used electrostatic method in MD simulations, and the highly parallel as well as GPU versions have been implemented in all major molecular dynamics packages such as AMBER, ${ }^{35} \mathrm{CHARMM}^{20}$ and GROMACS. ${ }^{17}$ With judicious use, i.e., avoiding small box size which may give rise to periodicity related artifacts ${ }^{36}$ and including counter ions to neutralize the simulation box, PME has enabled accurate simulations of not only soluble proteins, ${ }^{34}$ but also nucleic acids, ${ }^{37}$ lipid bilayers ${ }^{38}$ and transmembrane proteins. ${ }^{39}$

In this work we report the implementation of PME-based all-atom CpHMD in CHARMM. ${ }^{20}$ Test simulations employing titratable water, which we previously introduced to ensure the simulation system is charge neutral during titration, ${ }^{25}$ demonstrate significantly higher accuracy and faster convergence compared to the GRF-based all-atom CpHMD. The average absolute and maximum absolute errors of the calculated $\mathrm{p} K_{\mathrm{a}}$ 's for a stringent test set of proteins, HP36, BBL, HEWL and SNase, are 0.61 and 2.0 units with a sampling time of 10 ns per $\mathrm{pH}$ replica, as compared to the respective errors of 1.1 and 3.6 using the same amount of sampling by the GRF-based variant. Thus, PME-based all-atom CpHMD with titratable water is expected to become a general tool for conducting $\mathrm{pH}$-controlled molecular dynamics simulations.

\section{Methods and implementation}

\section{Continuous constant pH molecular dynamics}

CpHMD employs an extended Hamiltonian to simultaneously propagate spatial (real) and titration (virtual) coordinates, 6,7 
$\mathscr{H}\left(\left\{\mathbf{r}_{\mathbf{i}}\right\},\left\{\boldsymbol{\theta}_{\mathbf{t}}\right\}\right)=\sum_{\mathbf{i}}^{\mathbf{N}_{\text {atom }}} \frac{\mathbf{1}}{\mathbf{2}} \mathbf{m}_{\mathbf{i}} \dot{\mathbf{r}}_{\mathbf{i}}^{2}+\mathbf{U}^{\text {bond }}\left(\left\{\mathbf{r}_{\mathbf{i}}\right\}\right)+\mathbf{U}^{\text {nbond }}\left(\left\{\mathbf{r}_{\mathbf{i}}\right\},\left\{\boldsymbol{\theta}_{\mathbf{t}}\right\}\right)+\sum_{\mathbf{t}}^{\mathbf{N}_{\mathrm{titr}}} \frac{\mathbf{1}}{\mathbf{2}} \mathbf{m}_{\mathbf{t}} \dot{\theta}_{\mathbf{t}}^{\mathbf{2}}+\mathbf{U}^{*}\left(\boldsymbol{\theta}_{\mathbf{t}}\right)$,

where $i$ and $t$ refer to the atomic and titration-site index, respectively, $\mathbf{r}_{\mathbf{i}}$ represents the spatial coordinate, and $\theta_{t}$ is related to the titration coordinate $\lambda_{t}$ by $\lambda_{t}=\sin ^{2}\left(\theta_{t}\right)$. Thus, $\lambda_{t}$ is continuous and bound between 0 and 1 , corresponding to the protonated and deprotonated states, respectively. In practice, 0 and 1 are replaced with two cutoffs, $\lambda^{\mathrm{P}}$ and $\lambda^{\mathrm{U}}$, for defining the protonated and deprotonated states, respectively. Previous CpHMD studies $^{7-9,16,25,40,41}$ as well as current work show the calculated $\mathrm{p} K_{\mathrm{a}}$ 's are insensitive to the exact cutoffs, e.g., 0.1/0.9 or 0.2/0.8. This topic will be further discussed in Computational Details.

In Eq. 1, the first and the fourth terms give the kinetic energies of the real (atoms) and virtual $(\lambda)$ particles, respectively. The second term gives the titration-independent bonded energy. Note, although the change in bonded terms due to a switch in protonation state is small or negligible in current force fields, this is an approximation that will be eliminated in future work. The last term represents the biasing potential which is a sum of three terms,

$$
U^{*}\left(\boldsymbol{\theta}_{t}\right)=-U^{\mathrm{barr}}\left(\boldsymbol{\theta}_{t}\right)-U^{\bmod }\left(\boldsymbol{\theta}_{t}\right)+U^{\mathrm{pH}}\left(\boldsymbol{\theta}_{t}\right) .
$$

$U^{\text {barr }}$ a harmonic potential centered at 0.5 (midpoint of the $\lambda$ value range) to suppress the population of unphysical intermediate states,

$$
U^{\text {barr }}=4 \beta_{t}\left(\lambda_{t}-1 / 2\right)^{2}
$$

where $\beta_{t}$ is a parameter that specifies the barrier height and therefore controls the fraction of mixed states, i.e., $\lambda^{\mathrm{P}} \leq \lambda \leq \lambda^{\mathrm{U}}$. One $\beta$ value (typically about $2.0 \mathrm{kcal} / \mathrm{mol}$ ) is assigned for each model compound, e.g., Asp/Glu/His. This topic will be further discussed in Computational Details. $U^{\text {mod }}$ is the potential of mean force (PMF) function for deprotonation of the model compound, e.g., blocked amino acid in solution; and $U^{\mathrm{pH}}$ represent the free energy imposed on the deprotonation equilibrium to account for the deviation of the solution $\mathrm{pH}$ from the model $\mathrm{p} K_{\mathrm{a}}$ value,

$$
U^{\mathrm{pH}}\left(\lambda_{t}\right)=\ln (10) k_{b} T\left(p K_{a}^{\bmod }-\mathrm{pH}\right) \lambda_{t} .
$$

By invoking the linear response approximation, i.e., the charging free energy of an ion in polar solvent is quadratic in the charge perturbation and the charging potential is linear in the perturbation, ${ }^{42,43}$ the model PMF can be expressed as a quadratic function of $\lambda$, 


$$
U^{\bmod }\left(\lambda_{t}\right)=A_{t}\left(\lambda_{t}-B_{t}\right)^{2},
$$

where $A_{t}$ and $B_{t}$ are fitting parameters. It follows that the average force, $\langle\partial U / \partial \lambda\rangle$, is linear in $\lambda$. In practice, thermodynamic integration is applied to obtain the average forces at various $\lambda$ values, which are fit to a linear function to obtain $A_{t}$ and $B_{t}$. Note that linear response is formally exact when continuum solvation models are used for $\lambda$-dynamics, e.g., in implicit- ${ }^{6,7}$ and hybrid-solvent CpHMD. ${ }^{8}$ In fully explicit-solvent (all-atom) CpHMD, nonlinearity of solvent response is expected; however, surprisingly, in the GRF-based allatom CpHMD simulations with co-ions ${ }^{16}$ or titratable water, ${ }^{25}$ the degree of nonlinearity was found to be very small and negligible. Thus, the quadratic function will continue to be used in PME-CpHMD to fit the PMFs of model compounds and titratable water (hydronium/ hydroxide). Finally, we turn to the third term in Eq. 1, which represents the non-bond energy that depends on both spatial and titration coordinates, realizing the coupling between conformational dynamics and proton titration. Specifically, the van der Waals interactions involving titratable hydrogens and the partial charges on the titratable residues are linearly interpolated between the two end states. ${ }^{7}$ The latter results in a change in the electrostatic energy, which is the focus of the current development.

\section{Particle-mesh Ewald electrostatics}

Following the notation of Essmann et al., ${ }^{19}$ the electrostatic energy of $N$ point charges $\left\{q_{i}\right\}$ within a unit cell satisfying the condition $\sum_{i}^{N} q_{i}=0$ can be written as a lattice sum,

$$
U^{\text {elec }}=\frac{1}{2} \sum_{\mathbf{n}}^{\prime} \sum_{i} \sum_{j} \frac{q_{i} q_{j}}{\left|\mathbf{r}_{\mathbf{i}}-\mathbf{r}_{\mathbf{j}}+\mathbf{n}\right|}
$$

In the above equation the outer sum is over the vector $\mathbf{n}=\mathbf{n}_{\mathbf{1}} \mathbf{a}_{\mathbf{1}}+\mathbf{n}_{\mathbf{2}} \mathbf{a}_{2}+\mathbf{n}_{\mathbf{3}} \mathbf{a}_{3}$, where vectors $\mathbf{a}_{1}, \mathbf{a}_{2}$ and $\mathbf{a}_{3}$ are the edges of the unit cell; integers $n_{1}, n_{2}$ and $n_{3}$ indicate the location of the image. The prime indicates the summation excludes the term with $i=j$ and $n=0$ (selfinteractions).

In the particle-mesh Ewald (PME) method, the Coulomb lattice sum is decomposed into three terms, ${ }^{18,19}$

$$
U^{\text {elec }}=U^{\text {dir }}+U^{\text {rec }}+U^{\text {corr }} \text {. }
$$

The direct sum $U^{\mathrm{dir}}$ represents the short-range electrostatic energy calculated in real space, 


$$
U^{\operatorname{dir}}=\frac{1}{2} \sum_{\mathbf{n}}^{*} \sum_{i, j=1}^{N} \frac{q_{i} q_{j} \operatorname{erfc}\left(\beta\left|\mathbf{r}_{\mathbf{j}}-\mathbf{r}_{\mathbf{i}}+\mathbf{n}\right|\right)}{\left|\mathbf{r}_{\mathbf{j}}-\mathbf{r}_{\mathbf{i}}+\mathbf{n}\right|}
$$

where the asterisk denotes that self-interactions and those in the excluded list (for example, up to the second nearest neighbors) are omitted; erfc is the complementary error function; and $\beta$ is an arbitrary constant which determines the relative convergence rate of $U^{\text {dir }}$ and $U^{\text {rec }}$.

The reciprocal sum $U^{\text {rec }}$ represents the long-range electrostatics calculated in Fourier space as $^{18,19}$

$$
U^{\mathrm{rec}}=\frac{1}{2 \pi V} \sum_{\mathbf{m} \neq 0} \frac{\exp \left(-\pi^{2} \mathbf{m}^{2} / \beta^{2}\right)}{\mathbf{m}^{2}} S(\mathbf{m}) S(-\mathbf{m})
$$

Here $\mathbf{m}$ is the reciprocal lattice vector $\mathbf{m}=m_{1} \mathbf{a}_{1}^{*}+m_{2} \mathbf{a}_{2}^{*}+m_{3} \mathbf{a}_{3}^{*}$, where $m_{1}, m_{2}$ and $m_{3}$ are integers not all zero. $\mathbf{a}_{\alpha}^{*}$ is the conjugate reciprocal vector of $\mathbf{a}_{\alpha}$, which satisfies $\mathbf{a}_{\alpha}^{*} \cdot \mathbf{a}_{\beta}=\delta_{\alpha \beta}$, where $a, \beta=1,2,3 . V=\mathbf{a}_{1} \cdot \mathbf{a}_{2} \times \mathbf{a}_{3}$ refers to the volume of the unit cell, and $S(\mathbf{m})$ is the structure factor,

$$
S(\mathbf{m})=\sum_{j=1}^{N} q_{j} \exp \left(2 \pi i \mathbf{m} \cdot \mathbf{r}_{j}\right)
$$

The reciprocal term can be approximated as ${ }^{18,19}$

$$
U^{\mathrm{rec}}=\frac{1}{2} \sum_{m_{1}=0}^{K_{1}-1} \sum_{m_{2}=0}^{K_{2}-1} \sum_{m_{3}=0}^{K_{3}-1} Q\left(m_{1}, m_{2}, m_{3}\right) \cdot\left(\Theta_{\mathrm{rec}} \star Q\right)\left(m_{1}, m_{2}, m_{3}\right) .
$$

Here $K_{a}$, with $a=1,2,3$, is the size of reciprocal lattice. $Q$ is a three-dimensional array filled with charges on the reciprocal lattice grids. $\Theta_{\text {rec }}$ is the coefficient array independent of atomic charges. Convolution $\Theta_{\text {rec }} \star Q$ was achieved by one inverse and one forward threedimensional discrete fast Fourier transformations (3D-FFT). ${ }^{19}$

The correction term $U^{\text {corr }}$ is given as ${ }^{18,19,44}$

$$
U^{\mathrm{corr}}=-\frac{1}{2} \sum_{(i, j) \in M} \frac{q_{i} q_{j} \operatorname{erf}\left(\beta\left|\mathbf{r}_{\mathbf{i}}-\mathbf{r}_{\mathbf{j}}\right|\right)}{\left|\mathbf{r}_{\mathbf{i}}-\mathbf{r}_{\mathbf{j}}\right|}-\frac{\beta}{\sqrt{\pi}} \sum_{i=1}^{N} q_{i}^{2}-\frac{\pi}{2 \beta^{2} V}\left(\sum_{i} q_{i}\right)^{2} .
$$


Here the first term denotes the bonded pairs subtracted from $U^{\text {rec }}$, the second term represents the self energy, and the third term accounts for the interaction with a uniform background charge (plasma) included to neutralize the net charge of the system (if present). Note, in $U^{\text {eorr }}$ the dipole moment of the unit cell is neglected, i.e., conductive tinfoil boundary condition. Also, the last two terms are not evaluated in the atomic force calculations as they are independent of coordinates. Note that to obtain energies in the unit of $\mathrm{kcal} / \mathrm{mol}$, the electrostatic constant, $\boldsymbol{\kappa}=1 / 4 \pi \varepsilon_{0}=332.0 \mathrm{kcal} \cdot \AA \cdot \mathrm{mol}^{-1} \mathrm{e}^{-2}$, is multiplied to Eqs. $6,8,11$ and 12 .

Given the PME energies, electrostatic force on $\lambda$ particles can be obtained by differentiating the direct, reciprocal and correction terms with respect to $\lambda$ (subscript $t$ is omitted for clarity),

$$
\frac{\partial U^{\text {elec }}}{\partial \lambda}=\frac{\partial U^{\text {dir }}}{\partial \lambda}+\frac{\partial U^{\text {rec }}}{\partial \lambda}+\frac{\partial U^{\text {corr }}}{\partial \lambda},
$$

and realizing that $\lambda$ is linearly related to the atomic partial charges of the titratable group,

$$
q_{i}=\lambda q_{i}^{\mathrm{U}}+(1-\lambda) q_{i}^{\mathrm{P}} ; \frac{\partial q_{i}}{\partial \lambda}=q_{i}^{\mathrm{U}}-q_{i}^{\mathrm{P}} .
$$

In the above equations, $q_{i}$ represents the partial charge on atom $i$, and the superscript $\mathrm{U}$ and $\mathrm{P}$ denote the unprotonated and protonated states, respectively. While the $\lambda$ derivatives of $U^{\mathrm{dir}}$ and $U^{\text {corr }}$ are straightforward, the derivative of $U^{\text {rec }}$ can be written as

$$
\frac{\partial U^{\mathrm{rec}}}{\partial \lambda}=\sum_{m_{1}=0}^{K_{1}-1} \sum_{m_{2}=0}^{K_{2}-1} \sum_{m_{3}=0}^{K_{3}-1} \frac{\partial Q\left(m_{1}, m_{2}, m_{3}\right)}{\partial \lambda} \cdot\left(\Theta_{\mathrm{rec}} \star Q\right)\left(m_{1}, m_{2}, m_{3}\right) .
$$

Note, the factor 1/2 in Eq. 11 does not appear here because of the contribution from the convolution term where $Q$ is linear in $\lambda$.

\section{Correction of finite-size effects on $\mathrm{p} K_{\mathrm{a}}$ calculation}

It is well known that charging free energies calculated with lattice-sum methods under periodic boundary conditions are dependent on the periodic box size. ${ }^{45-47}$ The finite-size error has several physical origins, including the periodicity induced net charge interaction and undersolvation, discrete solvent effects, as well as residual integratd potential effects. ${ }^{47}$ Rocklin et al. recently showed that, for a system neutralized with counter-ions, the finite-size error in the charging free energy of a ligand (with +1 or -1 charge) is dominated by the discrete solvent effects due to an offset potential that compensates for the potential generated by discrete solvent. ${ }^{47}$ The corresponding offset energy in charging a titratable group is given as $^{47}$ 


$$
\Delta G^{\text {offset }}=\frac{2 \pi}{3} \kappa \gamma^{\text {solv }} Q \rho^{\text {solv }},
$$

where $x$ is the electrostatic constant, $\rho^{\text {solv }}$ is the solvent number density, $\rho^{\text {solv }}=N^{\text {sol }} / V$, where $N^{\text {solv }}$ and $V$ are the number of solvent molecules and volume of the periodic box, respectively. $Q$ is the charge of the titratable site, i.e., -1 for Asp/Glu or +1 for His/Lys, and $\gamma^{\text {solv }}$ is the quadrupole moment trace of the solvent model relative to a van der Waals interaction site. For a solvent model with a single van der Waals interaction site, e.g.,

$\mathrm{TIP} 3 \mathrm{P}, 48$ it is $\gamma^{\text {solv }}=\sum_{\mathrm{i}}^{n} q_{i} r_{i}^{2}$, where $\mathrm{n}$ is the number of atoms, $\mathrm{q}_{i}$ is the atomic partial charge, and $r_{i}$ is the distance to the van der Waals interaction site (oxygen). For TIP3P model $\gamma^{\text {solv }}$ is calculated as $0.764 \mathrm{e} \cdot \AA^{2}$.

From Eq. 16 we can obtain the difference in the offset potential between the protein and model compound simulations,

$$
\Delta \Delta G^{\text {offset }}=\frac{2 \pi}{3} \kappa \gamma^{\text {solv }} Q\left(\frac{N_{\text {prot }}^{\text {solv }}}{V_{\text {prot }}}-\frac{N_{\text {mod }}^{\text {solv }}}{V_{\text {mod }}}\right)
$$

where the subscripts prot and mod refer to the protein and model, respectively. In the model compound simulations, since the solute is very small compared to the simulation box, the solvent number density approaches that of the pure solvent $\rho^{\text {pure }}$, which is $0.0333679 \AA^{3}$ for water at ambient temperature and pressure. Combining Eq. 16 and 4, we obtain the corresponding $\mathrm{p} K_{\mathrm{a}}$ correction,

$$
\Delta p K_{a}^{\mathrm{corr}}= \pm \frac{\Delta \Delta G^{\mathrm{offset}}}{\ln (10) R T}
$$

where the negative sign is for acid groups and positive sign is for basic groups. Note, charging a basic group refers to the opposite of the deprotonation reaction. Thus, $\Delta \mathrm{p} K_{\mathrm{a}}{ }^{\text {corr }}$ is negative for both acidic and basic groups.

\section{Results and Discussion}

\section{Model parameterization and titration}

To validate the implementation, we first examine the PMF for model compound titration. It is expected that the GRF- and PME-based results are similar, while they are quite different from the hybrid-solvent simulation since the latter uses the GB model to calculate electrostatic forces along titration coordinates. Fig. 1 shows the average force obtained from thermodynamic integration and corresponding PMF for model Asp and Lys. It can be seen that linear fitting of the average force is perfect for hybrid-solvent simulations, while there is 
a small but negligible deviation for GRF- and PME-based simulations due to the use of explicit solvent (see earlier discussion). Thus, quadratic function is an accurate approximation of the PMF function for model compound titration. Comparing the GRF- and PME-based forces, we see that the deviation between the two becomes larger as $\lambda$ approaches 1 for Asp $\left(\mathrm{Asp}^{-}\right)$and 0 for Lys $\left(\mathrm{Lys}^{+}\right)$. This is because PME provides more stabilization for the charged state as compared to GRF, as shown in our previous study. ${ }^{30}$ Consequently, the free energy of deprotonation, i.e., charging for Asp and discharging for Lys, is larger in the PME-based simulation, consistent with our previous finding. ${ }^{30}$ It is worthwhile noting that the charging free energy for Asp and Lys (PMF difference between $\lambda$ 0 and 1) is very similar in the hybrid-solvent but not in the GRF- and PME-simulations. This is because the all-atom simulations are able to capture the phenomenon of charge asymmetry, attributable to the stronger interactions of anions with water, ${ }^{42,49}$ which is neglected in the GBSW implicit-solvent model.

Next we examine the titration simulations of model compounds using PME-based CpHMD in comparison to the hybrid-solvent and GRF-based CpHMD (Table 1). It is encouraging that, with $5 \mathrm{~ns}$ sampling per replica, the PME-based CpHMD is able to achieve a precision similar to experiment $\left(0.04-0.08\right.$ for the model alanine pentapeptide Ac-AA-X-AA- $\mathrm{NH}_{2}$ where $\mathrm{X}$ denotes the titratable residue).$^{50}$ This precision is on par with the hybrid-solvent CpHMD, which is known to deliver very fast convergence due to the use of the GB model for calculation of solvation forces along titration coordinates. ${ }^{8,9}$ Remarkably, the performance of the PME-based simulations is much better than that of the GRF-based simulations. With half of the sampling time per replica, random errors are reduced by more than half, which may be attributed to the energy fluctuation in the cutoff-based electrostatic methods. We note that, due to small fitting errors and perhaps nonlinearity in solvent response (see earlier discussion), there is often a small deviation between the calculated model $\mathrm{p} K_{\mathrm{a}}$ and reference value. In this work using the original fitting parameters, the deviations for Lys/Asp/Glu/His were 0, 0.1, 0.2 and 0.3, respectively. The deviations were then minimized using the iteration of titration simulation, $\mathrm{p} K_{\mathrm{a}}$ calculation and parameter adjustment (details see Table S5).

\section{Convergence of protein titration simulations}

We test the performance of PME-CpHMD using titration simulations of four proteins, HP36, BBL, HEWL and SNase. These proteins were chosen, since they have been used to benchmark the previous versions of CpHMD methods, ${ }^{8,25,40}$ and more importantly, they contain residues with large $\mathrm{p} K_{\mathrm{a}}$ shifts and coupled titration and as such serve as a stringent test for the accuracy of $\mathrm{p} K_{\mathrm{a}}$ calculations. ${ }^{53} \mathrm{The} \mathrm{p} K_{\mathrm{a}}$ 's of SNase are particularly challenging for the traditional PB-based electrostatic calculations and empirical $\mathrm{p} K_{\mathrm{a}}$ prediction methods, ${ }^{53-56}$ as there are many buried residues clustered together and titrating in a similar $\mathrm{pH}$ range (strong coupling).

We first examine the $\mathrm{p} K_{\mathrm{a}}$ convergence by monitoring the $\mathrm{p} K_{\mathrm{a}}$ 's cumulatively calculated as a function of simulation time (Fig. 2) and comparing the $\mathrm{p} K_{\mathrm{a}}$ values calculated with the first and second half of the 10-ns simulation. Compared to the GRF-based simulations, the convergence is much faster for all four proteins. Half of the $37 \mathrm{p} K_{\mathrm{a}}$ 's converge within $5 \mathrm{~ns}$ 
(per replica), defined as the difference between the first and second half of the simulation below 0.1 units. In contrast, only $7 \mathrm{p} K_{\mathrm{a}}$ 's become stable after $5 \mathrm{~ns}$ in the GRF-based simulations. At $10 \mathrm{~ns}$, most $\mathrm{p} K_{\mathrm{a}}$ 's are converged in the PME-based simulations but not in the GRF-based simulations.

To evaluate convergence, we also inspect the titration plots, i.e., fitting of the unprotonated fractions at different $\mathrm{pH}$ to the generalized Henderson-Hasselbalch $(\mathrm{HH})$ equation, as unconverged $\mathrm{p} K_{\mathrm{a}}$ 's typically manifest themselves in the "non-HH" behavior of the unprotonated fractions. As shown in Fig. 3, the fitting quality is excellent for all residues, with the chi-square value ranging from 0.00017 to 0.038 and correlation coefficient greater than 0.9955, even for Asp19 and Asp21 of SNase, which appear to have slightly larger fitting errors than other residues based on visual examination (see later discussion). These data are consistent with the overall good convergence. Finally, convergence of protonation state sampling can perhaps be best judged by examining the unprotonated fractions $(S)$ cumulatively calculated as a function of simulation time. This is because $S$ values are the direct representation of protonation state sampling and as such they are more sensitive than $\mathrm{p} K_{\mathrm{a}}$ values. Consistent with the calculated $\mathrm{p} K_{\mathrm{a}}$ 's vs. time (Figure 2), most $\mathrm{S}$ values converge after $10 \mathrm{~ns}$, except for Glu35/Asp48 of HEWL, and Asp19/Asp21/Glu75 of SNase, which are among those with large $\mathrm{p} K_{\mathrm{a}}$ errors (Supporting Information, Fig. S1-S4). However, encouragingly, the changes of $\mathrm{S}$ are all but one in the direction of decreasing the $\mathrm{p} K_{\mathrm{a}}$ deviations from experiment (see later for more discussion).

\section{Overall accuracy of protein titration simulations}

Next we examine the accuracy of the calculated $\mathrm{p} K_{\mathrm{a}}$ 's in comparison to the GRF-based as well as the hybrid-solvent CpHMD data (Table 2 and Fig. 4). The latter are used because hybrid-solvent CpHMD gives the most accurate $\mathrm{p} K_{\mathrm{a}}$ prediction thus far within the CpHMD framework. ${ }^{8,57}$ The overall average absolute and rms deviations from the PME-based simulations are respectively 0.61 and 0.77 , significantly lower than the GRF-based simulations (1.1 and 1.4), and similar to the hybrid-solvent data (0.59 and 0.76). Linear regression of the calculated $\mathrm{p} K_{\mathrm{a}}$ shifts (relative to model values) vs. experimental data gives a correlation coefficient of 0.79 for the PME-based simulations, significantly higher than the GRF-based (0.61) and hybrid-solvent data (0.70). Remarkably, the regression slope is about 1 and intercept is nearly 0 for the PME-based data, suggesting the absence of significant systematic errors. In contrast, the GRF-based data gives a sizable intercept of 0.77 , indicative of a systematic overestimation of $\mathrm{p} K_{\mathrm{a}}$ shifts, which may be attributed, in part to the overstabilization of neutral states by GRF as shown in our previous work, ${ }^{30}$ and in part to the lack of a finite-size correction as shown by Reif et al. in simulations based on the Baker-Watts reaction field. ${ }^{31,32}$ The hybrid-solvent data shows a slope well below one (0.61), indicative of a systematic underestimation of $\mathrm{p} K_{\mathrm{a}}$ shifts, which is due to the underestimation of desolvation penalty by the GBSW model ("too wet"). $8,9,57$ The abovementioned trend can also be seen from the histogram of $\mathrm{p} K_{\mathrm{a}}$ deviations, $\mathrm{p} K_{\mathrm{a}}{ }^{\text {calc }}-\mathrm{p} K_{\mathrm{a}}$ expt (Fig. S6). Accordingly, the histogram of hybrid-solvent simulations shows a larger population in the negative region, while the histogram of GRF simulations shows a larger population in the positive region. In contrast, the histogram of PME simulations is more or less symmetric around 0 . 


\section{Sources for the deviations between calculated and experimental $p K_{a}$ 's}

To understand the cause for the deviations between experimental and calculated $\mathrm{p} K_{\mathrm{a}}$ 's by PME-CpHMD, we identified 11 residues that show absolute errors above 0.6 units (Table 2, highlighted with asterisks): His142 (-1.1) and His166 (-1.3) of BBL; His15 (-1.5), Glu35 (1.0) and Asp52 (2.0) of HEWL; Asp19 (1.1), Asp40 (-1.0), Glu52 (0.8), Glu101 (0.9), Glu129 (1.7) and Glu135 (-0.8) of SNase. Among these residues, except for Asp40 and Glu135 of SNase, the calculated $\mathrm{p} K_{\mathrm{a}}$ 's for the acidic groups are too high, while those for histidines are too low, indicating that the neutral state was consistently overly favored in the simulation. We examine the structural environment and dynamical interactions involving these residues.

First, acidic residues that form persistent salt bridges show underestimated $\mathrm{p} K_{\mathrm{a}}$ 's to favor the charged state. For example, Asp40 and Glu135 of SNase form salt-bridge interactions with Arg35 and Arg105, respectively, leading to the underestimation of the $\mathrm{p} K_{\mathrm{a}}$ 's by 0.9 and 0.8 units, respectively. This type of error is also seen in the hybrid-solvent data (Table 2, underestimation of 0.8 and 0.4 units, respectively) as well as the previous GB-based CpHMD simulations, ${ }^{8}$ which can be reduced given more extensive sampling of the solventseparated configurations. ${ }^{8,40}$ In fact, the significant improvement (by 0.6 units) of the $\mathrm{p} K_{\mathrm{a}}$ of Asp48 from HEWL in the second $5 \mathrm{~ns}$ of the simulation is due to the weakened salt bridge with Arg61 (Fig. S7).

Second, (partially) buried residues that form persistent hydrogen bonds have the $\mathrm{p} K_{\mathrm{a}}$ 's overly shifted to favor the neutral state. For example, His 15 of HEWL forms persistent hydrogen bond with the hydroxyl group of Thr89, showing an overly downward-shifted $\mathrm{p} K_{\mathrm{a}}$ by 1.5 units. Glu129 of SNase is in persistent hydrogen bonding with the backbone amide group of Val111, showing a calculated $\mathrm{p} K_{\mathrm{a}}$ too high by 1.7 units. We suggest the overstabilization of the neutral state in these cases is due to the inadequate structural relaxation of the buried site, which results in an overestimation of the desolvation penalty. The same trend can be seen in the GRF-based data, although the overshifts there are exacerbated. Such overshifts are not seen in the hybrid-solvent simulations, which may be in part attributed to the fortuitous cancellation of errors between the underestimation of desolvation by the GBSW model and the overestimation of desolvation resulting from inadequate structural relaxation based on our previous studies of buried residues. ${ }^{9,57}$

The third group of residues with moderately large $\mathrm{p} K_{\mathrm{a}}$ errors (1-1.5 units) are those whose solvent accessibility is blocked by hydrophobic groups in direct contact. In order for CpHMD to reproduce experimental $\mathrm{p} K_{\mathrm{a}}$ 's of buried residues, our previous work showed that the ionization-induced increase in solvent exposure needs to be accurately captured in the simulation, which sometimes requires local unfolding. ${ }^{57}$ The latter aspect has been also demonstrated by the work of Warshel group ${ }^{58}$ and Garcia-Moreno group. ${ }^{59}$ In the PMEbased simulations, we found that H142, H166 of BBL and Glu101 of SNase are in persistent contact with Leu167, Leu144 and Leu124, respectively, preventing them from becoming fully exposed to solvent upon charging. As a result, the charged state is insufficiently stabilized, leading to the $\mathrm{p} K_{\mathrm{a}}$ underestimation by 1.1, 1.3 units for $\mathrm{H} 142, \mathrm{H} 166$ and overestimation by 0.9 units for Glu101. Note, the overestimated $\mathrm{p} K_{\mathrm{a}}$ shifts due to undersolvation of charged state can also be seen in the GRF-based results, although the deviation 
for Glu101 is much worse ( $K_{\mathrm{a}}$ is 2.5 units too high). The $\mathrm{p} K_{\mathrm{a}}$ overshifts are not seen in the hybrid-solvent simulations due to the error cancellation mentioned previously.

The last group of residues, Glu35 and Asp52 of HEWL and Asp19 and Asp21 of SNase, showing large $\mathrm{p} K_{\mathrm{a}}$ deviations from experiment are the so-called coupled residues, which are often found as catalytic dyad in enzyme active sites. with one elevated and one depressed $\mathrm{p} K_{\mathrm{a}}$ relative to the model value. In addition to desolvation and electrostatic repulsion, these residues may also be involved in hydrogen bonding and electrostatic interactions, making the $\mathrm{p} K_{\mathrm{a}}$ 's challenging to predict, as individual energetics needs to be modeled accurately. Our calculated $\mathrm{p} K_{\mathrm{a}}$ 's for Glu35 and Asp52 of HEWL are 7.1 and 5.6, respectively, in correct order but respectively 1.0 and 2.0 units higher than experiment (6.1 and 3.6). The calculated $\mathrm{p} K_{\mathrm{a}}$ shift of Asp52 has a wrong direction and carries the largest absolute error among all the calculated $\mathrm{p} K_{\mathrm{a}}$ 's. We suggest the error can be attributed to the aforementioned inadequate structure relaxation in the presence of persistent hydrogen bonding with the sidechain amide group of Asn59 in addition to the insufficient sampling of the coupled titration. The significant improvement (by 0.7 units) of the calculated $\mathrm{p} K_{\mathrm{a}}$ for Glu 35 in the second $5 \mathrm{~ns}$ of the simulation is due to a deprotonation-induced increase in the solvent exposure (Fig. S7).

The highly coupled $\mathrm{p} K_{\mathrm{a}}$ 's for Asp19 and Asp21 of SNase are notoriously difficult to predict due to hydrogen bonding between Asp19 and the backbone amide group of Asp21. In fact, in the blind $\mathrm{p} K_{\mathrm{a}}$ prediction exercise of $2009,{ }^{54}$ neither the empirical PROPKA, ${ }^{56}$ PoissonBoltzmann calculations ${ }^{55}$ or GBSW-based $\mathrm{CpHMD}^{9}$ were able to reproduce the experimental $\mathrm{p} K_{\mathrm{a}}$ order. Remarkably, our calculated $\mathrm{p} K_{\mathrm{a}}$ 's for Asp19 and Asp21 of SNase are 3.3 and 6.0, in correct order, although respectively 1.1 units too high and -0.5 units too low compared to experiment (2.2 and 6.5). The overestimation of the $\mathrm{p} K_{\mathrm{a}}$ of Asp19, which is the lower of the two catalytic $\mathrm{p} K_{\mathrm{a}}$ 's, is consistent with that of Asp52 in HEWL (calculation error of 2 units), and can be linked to the stable hydrogen bonding network with the sidechain hydroxyl, backbone amide of Thr22 and the backbone amide of Asp21. In addition to the correct order, the splitting of the two $\mathrm{p} K_{\mathrm{a}}$ 's (4.3) is well reproduced by the PME-based CpHMD (2.7), as compared to the GRF- (-0.5) and hybrid-solvent (1.2) simulations.

\section{Effect of prolonged sampling}

The above analysis suggests that inadequate sampling of structural relaxation accompanying the switch in protonation state is a major contributor to the deviations between experimental and calculated $\mathrm{p} K_{\mathrm{a}}$ 's with 10-ns sampling time. To investigate the sampling issue, which is particularly relevant for SNase where out of $17 \mathrm{Asp} / \mathrm{Glu}$ residues, 12 titrate in the $\mathrm{pH}$ range 3-4 (Fig. 3), we prolonged the simulations to $16 \mathrm{~ns}$ per replica. Time series of the unprotonated fractions and $\mathrm{p} K_{\mathrm{a}}$ 's of Asp19/Asp21/Glu75 that are not converged at $10 \mathrm{~ns}$ are converged after $12 \mathrm{~ns}$ (Fig. S4 and S5). To understand if the extra 6-ns sampling offers improvement in accuracy, we compare the $\mathrm{p} K_{\mathrm{a}}$ 's calculated based on 10-16 ns to those based on 5-10 sampling for the aforementioned residues with absolute deviations greater than 0.6, Asp19 (1.1), Asp40 (-1.0), Glu52 (0.8), Glu101 (0.9), Glu129 (1.7) and Glu135 $(-0.8)$. For Asp19, Asp21, Asp40 and Glu135, the deviations are decreased by 0.1, 0.3, 0.4 and 0.2, respectively; for Glu52 and Glu129 the deviations are increased by 0.2, 0.1; and for Glu101 the deviation is unchanged. Overall, the maximum, average and rmsd of the 
calculated $\mathrm{p} K_{\mathrm{a}}$ 's based on 10-16 ns are nearly the same as those based on 5-10 ns. These data suggest that while improvement is visible (for example, coupled residues Asp19 and Asp21), significant longer simulations may be needed in order to achieve overall improvement.

\section{Concluding remarks}

We developed the PME-based all-atom CpHMD and demonstrated the accuracy and convergence in protein titration simulations. Using $10 \mathrm{~ns}$ sampling per replica, the average absolute and rms errors of the calculated $\mathrm{p} K_{\mathrm{a}}$ 's are significantly lower than those from the GRF-CpHMD, and similar to the hybrid-solvent CpHMD (average absolute and RMS deviations are smaller by 0.01 ). The correlation with experimental $\mathrm{p} K_{\mathrm{a}}$ shifts are significantly improved over both GRF- and hybrid-solvent based simulations. Remarkably, the PME-based simulations give a linear regression slope of 1 and intercept near 0 , suggesting that systematic errors are largely absent.

Analysis showed that large deviations are from buried residues and those in stable hydrogen bond or salt bridge, which presents kinetic barrier to the configurations favored by the alternative protonation state. These data suggest that the $\mathrm{p} K_{\mathrm{a}}$ deviations observed in this work can be reduced given more extensive sampling of the ionization-induced change in solvent exposure as well as the breakage of hydrogen bond or salt bridge. Additional $6 \mathrm{~ns}$ sampling for SNase showed that, while some $\mathrm{p} K_{\mathrm{a}}$ 's are brought closer to experimental values, an overall improvement may require significantly longer simulations. Nevertheless, the agreement between the predicted and experimental $\mathrm{p} K_{\mathrm{a}}$ 's for the highly coupled catalytic dyad Asp19/Asp21 in SNase (in correct order and deviations of 1.1/-0.5 with $10 \mathrm{~ns}$ or $1.0 /-0.2$ with $16 \mathrm{~ns}$ simulations) is remarkable and suggests that the method may be deployed for the investigation of $\mathrm{pH}$-coupled conformational dynamics of enzymes.

We note a major limitation of the current implementation is speed. Compared to the conventional PME MD, PME-CpHMD adds about 30\% CPU time (based on the timing statistics of the four test proteins), similar to the hybrid-solvent and GRF-based CpHMD. However, we should note that current implementation is not optimized and is based on an MD engine CHARMM c36b2 which does not utilize the more recent parallelization methods. In principle, PME-based CpHMD adds only a fractional computational cost, because the number of titration coordinates is about two orders of magnitude smaller than the number of solute atoms. Another area of improvement is in the use of titratable water, ${ }^{16,25}$ which allows solute-solvent proton exchange and serves to maintain the total system charge neutral, an important part in PME-based simulations. ${ }^{45}$ However, for the latter purpose, alternatives such as the recently developed proton buffer method ${ }^{60}$ may be computationally more efficient. Thus, with the emergence of GPUs and new algorithms of parallel processing, we expect the integration of PME-based CpHMD in standard MD packages to enable $\mathrm{pH}$-controlled MD simulations on the order of hundreds of nano seconds on routine basis. As such we also anticipate a systematic improvement of accuracy in the calculated $\mathrm{p} K_{\mathrm{a}}$ 's. Finally, we shall emphasize that the PME-CpHMD can be applied to any system that current force fields can represent, thus opening a door to new insights into $\mathrm{pH}$ - 
dependent phenomena involving not only soluble proteins but also transmembrane proteins, nucleic acids, surfactants and polysaccharides.

\section{Computational Details}

\section{Preparation of simulation systems}

Model compounds are single amino acids (Asp, Glu, His and Lys) acetylated at N-terminus (ACE) and $\mathrm{N}$-methylamidated at $\mathrm{C}$-terminus $\left(\mathrm{CT}_{3}\right)$, hydronium $\left(\mathrm{H}_{3} \mathrm{O}^{+}\right)$and hydroxide $\left(\mathrm{OH}^{-}\right)$. The four test proteins are: the 36-residue subdomain of villin headpiece, HP36(PDB: 1VII), 45-residue binding domain of 2-oxoglutarate dehydrogenase multienzyme complex, BBL (PDB: 1W4H), 129-residue hen egg white lysozyme, HEWL (PDB: 2LZT), and 135residue hyperstable variant $(\Delta+\mathrm{PH})$ of staphylococcal nuclease (PDB: 3BDC). N-terminus of all proteins was left in the free and charged form, while the $\mathrm{C}$-terminus was blocked with CT3. The terminal groups were not made titratable, as they do not interact with the protein and often times no experimental $\mathrm{p} K_{\mathrm{a}}$ 's are available to compare with. However, this is an issue that will be addressed in our future work, perhaps by utilizing the model data obtained and collected by Pace group ${ }^{50,66}$ For protein structures, the crystal waters and hydrogens (if any) were stripped and the HBUILD function in CHARMM was used to add hydrogen atoms. Model compounds were constructed using CHARMM. ${ }^{20}$ The structure was solvated in a box (cubic for model compounds and truncated octahedron for proteins) filled with the CHARMM modified TIP3P water ${ }^{20}$ with a distance of at least $10 \AA$ between the solute and edges of the box. The unit-cell lattice parameters are: 30 (model compounds), 54 (HP36), 59 (BBL), 69 (HEWL) and $70 \AA$ (SNase). To test the finite-size effects and corrections, additional box sizes were considered for the two smaller proteins HP36 (43 A) and BBL (49 $\AA$ ).

To maintain a constant net charge for the system, $N_{\text {titr }}$ randomly chosen TIP3P water was replaced with titratable water that can convert to hydroxide or hydronium. ${ }^{25}$ For simplicity, we refer to the former titratable water as hydroxide and the latter as hydronium. For model compounds, 1 hydroxide or hydronium was added for the titration of model Asp/Glu or model His/Lys. For proteins, 4 hydroxide were added for HP36, 2 hydronium/6 hydroxide for BBL, 1 hydronium/9 hydroxide for HEWL, and 2 hydronium/17 hydroxide for SNase. To neutralize the simulation box, $\mathrm{Na}^{+}$or $\mathrm{Cl}^{-}$counterions were then added. For proteins, additional salt ions were added to reach the experimental ionic strength: $150 \mathrm{mM}$ for HP36, ${ }^{67} 200 \mathrm{mM}$ for BBL, ${ }^{62,63} 50 \mathrm{mM}$ for HEWL, ${ }^{64}$ and 100mM for SNASE. ${ }^{65}$ Thus, the model compound simulations contained $1 \mathrm{Na}^{+}$for Asp/Glu and $1 \mathrm{Cl}^{-}$for His/Lys, while the protein simulations contained $10 \mathrm{Na}^{+} / 13 \mathrm{Cl}^{-}$for $\mathrm{HP} 36,16 \mathrm{Na}^{+} / 20 \mathrm{Cl}^{-}$for BBL, $2 \mathrm{Na}^{+} / 12$ $\mathrm{Cl}^{-}$for $\mathrm{HEWL}$, and $15 \mathrm{Na}^{+} / 24 \mathrm{Cl}^{-}$for SNASE.

\section{Simulation protocol}

The method described in this work was implemented in an inhouse modified version of CHARMM (release c36b2). ${ }^{20}$ Particle mesh Ewald (PME) method was added to the PHMD module to account for the electrostatic force on the fictitious $\lambda$-particles. The CHARMM22/ CMAP additive force field was used to represent the proteins and the CHARMM modified 
TIP3P water model was used to represent nontitratable solvent. The force field parameters for titratable water are taken from our previous work. ${ }^{25}$

All simulations were performed using the $\mathrm{pH}$ replica-exchange (pH-REX) protocol, ${ }^{8}$ where each replica underwent constant NPTpH molecular dynamics (MD) at $300 \mathrm{~K}, 1 \mathrm{~atm}$ pressure and specified $\mathrm{pH}$. The temperature, pressure and $\mathrm{pH}$ were controlled by Hoover thermostat, ${ }^{68}$ Langevin piston pressure coupling algorithm, ${ }^{69}$ and $\mathrm{CpHMD},{ }^{6,7}$ respectively. Periodic boundary condition was applied to all simulations. The SHAKE algorithm was applied to all bonds involving hydrogen atoms to allow a 2-fs integration time step. In the PME calculation, the real-space cutoff was $12 \AA$ and grid spacing was $1 \AA$. The van der Waals energies and forces were smoothly turned off over the range of $10-12 \AA$ via a switching function. The titration $(\lambda)$ coordinates were propagated using the Langevin algorithm with a collision frequency of $5 \mathrm{ps}^{-1}$ (default setting in PHMD). The mass of the $\lambda$ particles was set to 10 atomic mass units (default setting in PHMD). The parameter $\beta$ used in $U^{\text {barr }}$ was set to $2.0 \mathrm{kcal} / \mathrm{mol}$ for all titratable residues (see Table S5).

For derivation of model PMF functions, thermodynamic integration was performed using 1ns CPT MD at $\theta$ values of $0.2,0.4,0.6,0.7854,1.0,1.2$, and 1.4. The second half of the trajectory was used to determine the average force $\langle\partial U / \partial \theta\rangle$. Five independent $\mathrm{pH}$-REX simulations were conducted for each model compound. Each pH-REX simulation utilized five $\mathrm{pH}$ replicas occupying the $\mathrm{pH}$ conditions of $\mathrm{p} K_{\mathrm{a}}{ }^{\text {ref }}-2, \mathrm{p} K_{\mathrm{a}}{ }^{\text {ref }}-1, \mathrm{p} K_{\mathrm{a}}{ }^{\text {ref }}, \mathrm{p} K_{\mathrm{a}}{ }^{\text {ref }}+1$, $\mathrm{p} K_{\mathrm{a}}{ }^{\text {ref }}+2$, where $\mathrm{p} K_{\mathrm{a}}{ }^{\text {ref }}$ refers to the reference model $\mathrm{p} K_{\mathrm{a}}$ value. Each $\mathrm{pH}$ replica was simulated for $5 \mathrm{~ns}$.

Prior to simulations energy minimization was performed using steepest descent (SD) and adoptive basis Newton-Ralphson (ABNR) methods. Three rounds of minimization each consisting of 50 steps SD followed by 50 steps ABNR were performed. Finally, 100 steps of SD followed by 100 steps of ABNR minimization were performed. Protein simulations began with a heating stage followed by equilibration at $\mathrm{pH} 7$ before invoking $\mathrm{pH}-\mathrm{REX}$ simulations. In the heating stage, the desired temperature of $300 \mathrm{~K}$ was achieved in $120 \mathrm{ps}$ with the heavy atoms harmonically restrained by a force constant of $5.0 \mathrm{kcal} / \mathrm{mol} \cdot \AA$. In the equilibration stage, two 20-ps simulations were performed with the force constants of 1.0 and $0.1 \mathrm{kcal} / \mathrm{mol} \cdot \AA$, respectively, followed by a 20 -ps unrestrained MD.

In the $\mathrm{pH}-\mathrm{REX}$ simulation, identical starting structure was used in all $\mathrm{pH}$ replicas and unless otherwise noted each replica was simulated for $10 \mathrm{~ns}$. The $\mathrm{pH}$ spacing was $0.5 \mathrm{pH}$ unit and the $\mathrm{pH}$ range extended at least 1 unit above and below the highest and lowest experimental $\mathrm{p} K_{\mathrm{a}}$ values. Specifically, the $\mathrm{pH}$ range was 1 to 6.5 for HP36, 0.5 to 8.0 for BBL, -1.0 to 9.5 for HEWL and SNase. An exchange between adjacent $\mathrm{pH}$ replicas was attempted every 100 MD steps or $0.2 \mathrm{ps}$ in model compound titration and 500 steps or $1 \mathrm{ps}$ in protein simulations. The average replica-exchange ratio was above $40 \%$. In the simulations of HP36, BBL and HEWL, most replicas walked through the entire $\mathrm{pH}$ range, while in the simulation of SNase, only half of the replicas did so (Fig. S8-S11). 


\section{$\mathrm{p} K_{\mathrm{a}}$ calculation}

Based on the $\lambda$ values, which were collected after each exchange step, the unprotonated fractions $(S)$ were calculated using the same two cutoffs $(0.9 / 0.1)$ as in the previous versions of CpHMD, ${ }^{6-8,16,25,40}$

$$
S=\frac{N^{\mathrm{U}}}{N^{\mathrm{P}}+N^{\mathrm{U}}}
$$

Here $N^{\mathrm{U}}$ and $N^{P}$ represent the number of deprotonated $(\lambda>0.9)$ and protonated $(\lambda<0.1)$ states, respectively. The $\mathrm{p} K_{\mathrm{a}}$ was then calculated by fitting $S$ at simulated $\mathrm{pH}$ conditions to the generalized Henderson-Hasselbalch or Hill equation,

$$
S=\frac{1}{1+10^{n\left(\mathrm{p} K_{a}-\mathrm{pH}\right)}},
$$

where $n$ is the Hill coefficient.

We compared the $\mathrm{p} K_{\mathrm{a}}$ 's calculated using two sets of $\lambda$ cutoffs: 0.1/0.9 and 0.2/0.8 (see Table S6). Most $\mathrm{p} K_{\mathrm{a}}$ 's are the same; the largest difference is 0.1 ; the overall comparison with experiment is also very similar. The RMSD, regression slope and intercept from the calculations using the $0.1 / 0.9$ cutoffs are $0.77,0.98$ and 0.06 , while the corresponding values with the $0.2 / 0.8$ cutoffs are $0.76,1.01$ and 0.11 . This behavior is consistent with the previous versions of CpHMD. ${ }^{6-8,16,25,40}$ With the two sets of cutoffs, the fraction of mixed states is in the range of $0.1-0.45$ and $0.05-0.3$, respectively. Very low fractions of mixed states are typically found at low and high $\mathrm{pH}$ conditions, whereas higher fractions are found around the titration $\mathrm{pH}$. We note that, given a set of cutoffs such as $0.1 / 0.9$, the parameter $\beta$ that controls the barrier height can be adjusted to achieve a desired fraction of mixed states, taking into account higher barrier height leads to slower $\lambda$ transition. Optimization of the $\beta$ parameter will be a topic in our future work.

\section{Finite-size correction of protein $\mathrm{p} K_{\mathrm{a}}$ 's}

We applied Eqs. 17 and 18 to calculate the $\mathrm{p} K_{\mathrm{a}}$ corrections to account for the box size dependence. It follows that the corrections are: -0.5 for Asp/Glu of HP36; -0.5 for Asp and -0.4 for Glu/His of BBL; -0.9 for Asp/Glu and -0.8 for His of HEWL; and -0.9 for Asp/Glu/His of SNase. These corrections are very similar to those pre-calculated using the lattice-parameter-based volume of the protein system (Table S1 and S2). Thus, the $\mathrm{p} K_{\mathrm{a}}$ corrections will be incorporated into the reference $\mathrm{p} K_{\mathrm{a}}$ 's and implemented in the CpHMD code in the future. More details are given in SI. Finally, to test the finite-size effects and validate the $\mathrm{p} K_{\mathrm{a}}$ corrections, we performed additional simulations for two small proteins, HP36 and BBL, using a smaller box. The differences in the $\mathrm{p} K_{\mathrm{a}}$ 's are very similar to those estimated using the finite-size corrections (Table S3). 


\section{Supplementary Material}

Refer to Web version on PubMed Central for supplementary material.

\section{Acknowledgments}

Financial support is provided by National Science Foundation (MCB1305560 and CBET1435957) and National Institutes of Health (GM098818). We thank David Mobley and Gabriel Rocklin for pointing out the finite-size effect.

\section{References}

1. Baptista AM, Teixeira VH, Soares CM. Constant-pH Molecular Dynamics using Stochastic Titration. J Chem Phys. 2002; 117:4184-4200.

2. Mongan J, Case DA, McCammon JA. Constant pH Molecular Dynamics in Generalized Born Implicit Solvent. J Comput Chem. 2004; 25:2038-2048. [PubMed: 15481090]

3. Swails JM, York DM, Roitberg AE. Constant pH Replica Exchange Molecular Dynamics in Explicit Solvent Using Discrete Protonation States: Implementation, Testing, and Validation. J Chem Theory Comput. 2014; 10:1341-1352. [PubMed: 24803862]

4. Lee J, Miller BT, Damjanović A, Brooks BR. Constant pH Molecular Dynamics in Explicit Solvent with Enveloping Distribution Sampling and Hamiltonian Exchange. J Chem Theory Comput. 2014; 10:2738-2750. [PubMed: 25061443]

5. Kong X, Brooks CL III. $\lambda$-dynamics: A New Approach to Free Energy Calculations. J Chem Phys. 1996; 105:2414-2423.

6. Lee MS, Salsbury FR Jr, Brooks CL III. Constant-pH Molecular Dynamics using Continuous Titration Coordinates. Proteins. 2004; 56:738-752. [PubMed: 15281127]

7. Khandogin J, Brooks CL III. Constant pH Molecular Dynamics with Proton Tautomerism. Biophys J. 2005; 89:141-157. [PubMed: 15863480]

8. Wallace JA, Shen JK. Continuous Constant pH Molecular Dynamics in Explicit Solvent with pHbased Replica Exchange. J Chem Theory Comput. 2011; 7:2617-2629. [PubMed: 26606635]

9. Wallace JA, Wang Y, Shi C, Pastoor KJ, Nguyen BL, Xia K, Shen JK. Toward Accurate Prediction of $\mathrm{p} K_{a}$ Values for Internal Protein Residues: the Importance of Conformational Relaxation and Desolvation Energy. Proteins. 2011; 79:3364-3373. [PubMed: 21748801]

10. TDM, Edwards J, Roitberg AE. pH-REMD Simulations Indicate that the Catalytic Aspartates of HIV-1 Protease Exist Primarily in a Monoprotonated State. J Phys Chem B. 2014; 118:1257712585. [PubMed: 25340507]

11. Morrow BH, Wang Y, Wallace JA, Koenig PH, Shen JK. Simulating pH Titration of a Single Surfactant in Ionic And Nonionic Surfactant Micelles. J Phys Chem B. 2011; 115:14980-14990. [PubMed: 22050243]

12. Vila-Viçosa D, Teixeira VH, Santos HAF, Baptista AM, Machuqueiro M. Treatment of Ionic Strength in Biomolecular Simulations of Charged Lipid Bilayers. J Chem Theory Comput. 2014; 10:5483-5492. [PubMed: 26583231]

13. Chen W, Morrow BH, Shi C, Shen JK. Recent Development and Application of Constant pH Molecular Dynamics. Mol Simulat. 2014; 40:830-838.

14. Donnini S, Tegeler F, Groenhof G, Grubmüller H. Constant pH Molecular Dynamics in Explicit Solvent with $\lambda$-dynamics. J Chem Theory Comput. 2011; 7:1962-1978. [PubMed: 21687785]

15. Goh GB, Knight JL, Brooks CL III. Constant pH Molecular Dynamics Simulations of Nucleic Acids in Explicit Solvent. J Chem Theory Comput. 2012; 8:36-46. [PubMed: 22337595]

16. Wallace JA, Shen JK. Charge-leveling and Proper Treatment of Long-range Electrostatics in AllAtom Molecular Dynamics at Constant pH. J Chem Phys. 2012; 137:184105. [PubMed: 23163362]

17. Pronk S, Páll S, Schulz R, Larsson P, Bjelkmar P, Apostolov R, Shirts MR, Smith JC, Kasson PM, van der Spoel D, Hess B, Lindahl E. GROMACS 4. 5: A High-Throughput and Highly Parallel 
Open Source Molecular Simulation Toolkit. Bioinformatics. 2013; 29:845-854. [PubMed: 23407358]

18. Darden T, York D, Pedersen L. Particle mesh Ewald: An Nlog(N) method for Ewald Sums in Large Systems. J Chem Phys. 1993; 98:10089-10092.

19. Essmann U, Perera L, Berkowitz ML, Darden T, Hsing L, Pedersen LG. A Smooth Particle Mesh Ewald Method. J Chem Phys. 1995; 103:8577-8593.

20. Brooks BR, Brooks CL III, Mackerell AD Jr, Nilsson L, Petrella RJ, Roux B, Won Y, Archontis G, Bartles C, Boresch S, Caflisch A, Caves L, Cui Q, Dinner AR, Feig M, Fischer S, Gao J, Hodoscek M, Im W, Lazaridis KKT, Ma J, Ovchinnikov V, Paci E, Pastor RW, Post CB, Pu JZ, Schaefer M, Tidor B, Venable RM, Woodcock HL, Wu X, Yang W, York DM, Karplus M. CHARMM: the Biomolecular Simulation Program. J Comput Chem. 2009; 30:1545-1614. [PubMed: 19444816]

21. Goh GB, Knight JL, Brooks CL. pH-Dependent Dynamics of Complex RNA Macromolecules. J Chem Theory Comput. 2013; 9:935-943. [PubMed: 23525495]

22. Goh GB, Hulbert BS, Zhou H III, CLB. Constant pH Molecular Dynamics of Proteins in Explicit Solvent with Proton Tautomerism. Proteins. 2014; 82:1319-1331. [PubMed: 24375620]

23. Panahi A, Charles L, Brooks I. Membrane Environment Modulates the $\mathrm{p} K_{a}$ Values of Transmembrane Helices. J Phys Chem B. 2015; 119:4601-4607. [PubMed: 25734901]

24. Tironi IG, Sperb R, Smith PE, van Gunsteren WF. A Generalized Reaction Field Method for Molecular Dynamics Simulations. J Chem Phys. 1995; 102:5451-5459.

25. Chen W, Wallace J, Yue Z, Shen J. Introducing Titratable Water to All-Atom Molecular Dynamics at Constant pH. Biophys J. 2013; 105:L15-L17. [PubMed: 23972860]

26. Morrow BH, Eike DM, Murch BP, Koenig PH, Shen JK. Predicting Proton Titration in Cationic Micelle and Bilayer Environments. J Chem Phys. 2014; 141:084714. [PubMed: 25173037]

27. Morrow BH, Payne GF, Shen J. pH-Responsive Self-Assembly of Polysaccharide Through a Rugged Energy Landscape. J Am Chem Soc. 2015; 137:13024-13030. [PubMed: 26383701]

28. Barker JA, Watts RO. Monte Carlo Studies of the Dielectric Properties of Water-Like Models. Mol Phys. 1973; 26:789-792.

29. Klauda JB, Venable RM, Freites JA, OConnor JW, Tobias DJ, Mondragon-Ramirez C, Vorobyov I, MacKerell Alexander D Jr, Pastor RW. Update of the CHARMM All-Atom Additive Force Field for Lipids: Validation on Six Lipid Types. J Phys Chem B. 2010; 114:7830-7843. [PubMed: 20496934]

30. Chen W, Shen JK. Effects of System Net Charge and Electrostatic Truncation on All-Atom Constant pH Molecular Dynamics. J Comput Chem. 2014; 35:1986-1996. [PubMed: 25142416]

31. Reif MM, Hünenberger PH, Oostenbrink C. New interaction Parameters for Charged Amino Acid Side Chains in the GROMOS Force Field. J Chem Theory Comput. 2012; 8:3705-3723. [PubMed: 26593015]

32. Reif MM, Oostenbrink C. Net Charge Changes in the Calculation of Relative Ligand-Binding Free Energies via Classical Atomistic Molecular Dynamics Simulation. J Comput Chem. 2014; 35:227243. [PubMed: 24249099]

33. Gargallo R, Hünenberger PH, Avilés FX, Oliva B. Molecular Dynamics Simulation of Highly Charged Proteins: Comparison of the Particle-Particle Particle-Mesh and Reaction Field Methods for the Calculation of Electrostatic Interactions. Protein Sci. 2003; 12:2161-2172. [PubMed: 14500874]

34. Lange OF, van der Spoel D, de Groot BL. Scrutinizing Molecular Mechanics Force Fields on the Submicrosecond Timescale with NMR Data. Biophys J. 2010; 99:647-655. [PubMed: 20643085]

35. Case, D., Betz, R., Botello-Smith, W., Cerutti, D., Cheatham, T., III, Darden, T., Duke, R., Giese, T., Gohlke, H., Goetz, A., Homeyer, N., Izadi, S., Janowski, P., Kaus, J., Kovalenko, A., Lee, T., LeGrand, S., Li, P., Lin, C., Luchko, T., Luo, R., Madej, B., Mermelstein, D., Merz, K., Monard, G., Nguyen, H., Nguyen, H., Omelyan, I., Onufriev, A., Roe, D., Roitberg, A., Sagui, C., Simmerling, C., Swails, J., Walker, R., Wang, J., Wolf, R., Wu, X., Xiao, L., York, D., Kollman, P. AMBER 2016. University of California; San Francisco: 2016.

J Chem Theory Comput. Author manuscript; available in PMC 2017 December 04. 
36. Hünenberger PH, McCammon JA. Effect of Artificial Periodicity in Simulations of Biomolecules under Ewald Boundary Conditions: A Continuum Electrostatics Study. Biophys Chem. 1999; 78:69-88. [PubMed: 10343384]

37. Cheatham TE III, Kollman PA. Molecular Dynamics Simulation of Nucleic Acids. Annu Rev Phys Chem. 2000; 51:435-471. [PubMed: 11031289]

38. Patra M, Karttunen M, Hyvönen MT, Falck E, Lindqvist P, Vattulainen I. Molecular Dynamics Simulations of Lipid Bilayers: Major Artifacts due to Truncating Electrostatic Interactions. Biophys J. 2003; 84:3636-3645. [PubMed: 12770872]

39. Ash WL, Zlomislic MR, Oloo EO, Tieleman DP. Computer simulations of membrane proteins. Biochim Biophys Acta. 2004; 1666:158-189. [PubMed: 15519314]

40. Khandogin J, Brooks CL III. Toward the Accurate First-Principles Prediction of Ionization Equilibria in Proteins. Biochemistry. 2006; 45:9363-9373. [PubMed: 16878971]

41. Wallace JA, Shen JK. Predicting $\mathrm{p} K_{a}$ Values with Continuous Constant $\mathrm{pH}$ Molecular Dynamics. Methods Enzymol. 2009; 466:455-475. [PubMed: 21609872]

42. Levy RM, Gallicchio E. Computer Simulations with Explicit Solvent: Recent Progress in the Thermodynamic Decomposition of Free Energies and in Modeling Electrostatic Effects. Annu Rev Phys Chem. 1998; 49:531-567. [PubMed: 9933909]

43. Simonson T. Gaussian Fluctuations And Linear Response in an Electron Transfer Protein. Proc Natl Acad Sci USA. 2001; 99:6544-6549.

44. Figueirido F, Del Buono GS, Levy RM. On Finitie-Size Effects in Computer Simulations using Ewald Potential. J Chem Phys. 1995; 103:6133-6142.

45. Hünenberger PH, McCammon JA. Ewald Artifacts in Computer Simulations of Ionic Solvation and Ion-Ion Interaction: A Continuum Electrostatics Study. J Chem Phys. 1999; 110:1856-1872.

46. Kastenholz MA, Hünenberger PH. Influence of Artificial Periodicity and Ionic Strength in Molecular Dynamics Simulations of Charged Biomoleculaes Employing Lattice-Sum Methods. J Phys Chem B. 2004; 108:774-788.

47. Rocklin GJ, Mobley DL, Dill KA, Hünenberger PH. Calculating the Binding Free Energies of Charged Species Based on Explicit-Solvent Simulations Employing Lattice-Sum Methods: An Accurate Correction Scheme for Electrostatic Finite-Size Effects. J Chem Phys. 2013; 139:184103. [PubMed: 24320250]

48. Jorgensen WL, Chandrasekhar J, Madura JD, Impey RW, Klein ML. Comparison of Simple Potential Functions for Simulating Liquid Water. J Chem Phys. 1983; 79:926-935.

49. Hummer G, Pratt LR, García AE. Free energy of ionic hydration. J Phys Chem. 1996; 100:12061215.

50. Thurlkill RL, Grimsley GR, Scholtz JM, Pace CN. pK alues of the Ionizable Groups of Proteins. Protein Sci. 2006; 15:1214-1218. [PubMed: 16597822]

51. Nozaki Y, Tanford C. Examination of titration behavior. Methods Enzymol. 1967; 11:715-734.

52. Bashford D, Case DA, Dalvit C, Tennant L, Wright PE. Electrostatic Calculations of Side-Chain $\mathrm{p} K_{a}$ a Values in Myoglobin and Comparison with NMR Data for Histidines. Biochemistry. 1993; 32:8045-8056. [PubMed: 8347606]

53. Schutz CN, Warshel A. What Are the Dielectric Constants of Proteins and How to Validate Electrostatic Models? Proteins. 2001; 44:400-417. [PubMed: 11484218]

54. Alexov E, Mehler EL, Baker N, Baptista AM, Huang Y, Milletti F, Nielsen JE, Farrell D, Carstensen T, Olsson MHM, Shen JK, Warwicker J, Williams S, Word JM. Progress in the Prediction of $\mathrm{p} K_{a}$ Values in Proteins. Proteins. 2011; 79:3260-3275. [PubMed: 22002859]

55. Gunner MR, Zhu X, Klein MC. MCCE analysis of the $\mathrm{p} K_{a}$ s of Introduced Buried Acids and Bases in Staphylococcal Nuclease. Proteins. 2011; 79:3306-3319. [PubMed: 21910138]

56. Olsson MHM. Protein Electrostatics and $\mathrm{p} K_{a}$ Blind Predictions; Contribution from Empirical Predictions of Internal Ionizable Residues. Proteins. 2011; 79:3333-3345. [PubMed: 22072518]

57. Shi C, Wallace JA, Shen JK. Thermodynamic Coupling of Protonation and Conformational Equilibria in Proteins: Theory And Simulation. Biophys J. 2012; 102:1590-1597. [PubMed: 22500759] 
58. Kato M, Warshel A. Using a Charging Coordinate in Studies of Ionization Induced Partial Unfolding. J Phys Chem B. 2006; 110:11566-11570. [PubMed: 16771433]

59. Damjanović A, Brooks BR, García-Moreno EB. Conformational Relaxation and Water Penetration Coupled to Ionization of Internal Groups in Proteins. J Phys Chem A. 2011; 115:4042-4053. [PubMed: 21428436]

60. Donnini S, Ullmann RT, Groenhof G, Grubmüller H. Charge-Neutral Constant pH Molecular Dynamics Simulations Using a Parsimonious Proton Buffer. J Chem Theory Comput. 2016; 12:1040-1051. [PubMed: 26881315]

61. Xiao S, Patsalo V, Shan B, Bi Y, Green DF, Raleigh DP. Rational Modification of Protein Stability by Targeting Surface Sites Leads to Complicated Results. Proc Natl Acad Sci USA. 2013; 110:11337-11342. [PubMed: 23798426]

62. Arbely E, Rutherford TJ, Sharpe TD, Ferguson N, Fersht AR. Downhill versus Barrier-Limited Folding of BBL: Energetic and Structural Pertubation Effects upon Protonation of a Histidine of Unusually low $\mathrm{p} K_{a}$. J Mol Biol. 2009; 387:986-992. [PubMed: 19136007]

63. Arbely E, Rutherford TJ, Neuweiler H, Sharpe TD, Ferguson N, Fersht AR. Carboxyl p $K_{a}$ Values and Acid Denaturation of BBL. J Mol Biol. 2010; 403:313-327. [PubMed: 20816989]

64. Webb H, Tynan-Connolly BM, Lee GM, Farrell D, O’Meara F, Søndergaard CR, Teilum K, Hewage C, McIntosh LP, Nielsen JE. Remeasuring HEWL $\mathrm{p} K_{a}$ Values by NMR Spectroscopy: Methods, Analysis, Accuracy, and Implications for Theoretical p $K_{a}$ Calculations. Proteins. 2011; 79:685-702. [PubMed: 21287606]

65. Castañeda CA, Fitch CA, Majumdar A, Khangulov V, Schlessman JL, García-Moreno EB. Molecular Determinants of the $\mathrm{p} K_{a}$ Values of Asp and Glu Residues in Staphylococcal Nuclease. Proteins. 2009; 77:570-588. [PubMed: 19533744]

66. Grimsley GR, Scholtz JM, Pace CN. A Summary of the Measured pK Values of the Ionizable Groups in Folded Proteins. Protein Sci. 2009; 18:247-251. [PubMed: 19177368]

67. Bi, Y. PhD thesis. Stony Brook University; 2008. Studies of the Folding and Stability of the Villin Headpiece Subdomain.

68. Hoover WG. Canonical Dynamics: Equilibration Phase-Space Distributions. Phys Rev A. 1985; 31:1695-1697.

69. Feller SE, Zhang Y, Pastor RW, Brooks BR. Constant pressure molecular dynamics simulation: The Langevin piston method. J Chem Phys. 1995; 103:4613-4621. 

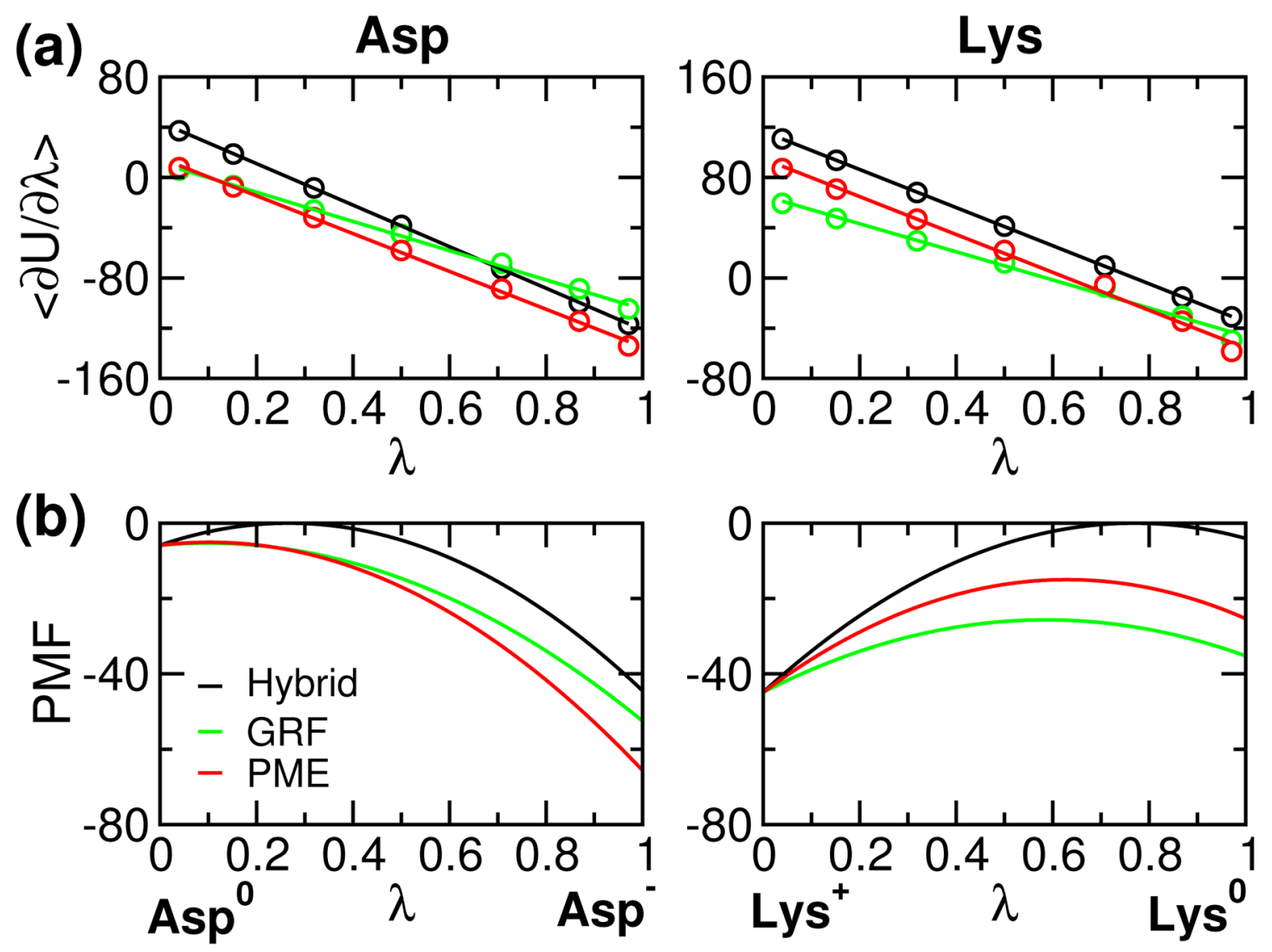

Figure 1.

Average force and corresponding potential of mean force (PMF) for deprotonation of model compounds Asp and Lys. (a) Average force $\langle\partial U / \partial \lambda\rangle$ calculated at different values of $\lambda$. The average force was obtained from the second half of the 1-ns thermodynamic Integration (SI). Lines are the best fits to a linear function $2 A(\lambda-B$ ) (see Eq. 5). (b) Corresponding PMF function of $\lambda$ (integration of the average force). In (a) and (b), data from the hybrid-solvent, ${ }^{8}$ GRF-25 and PME-based CpHMD simulations are shown in black, green and red, respectively. The unit of force or PMF is $\mathrm{kcal} / \mathrm{mol}$. 
(a)

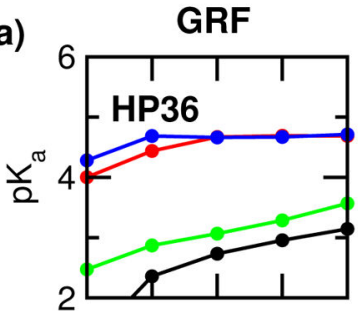

(b)

(c)
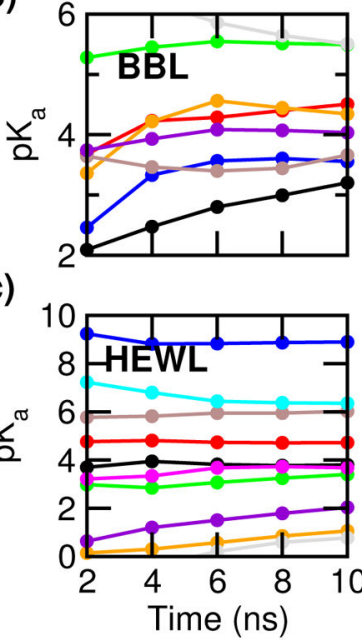

PME
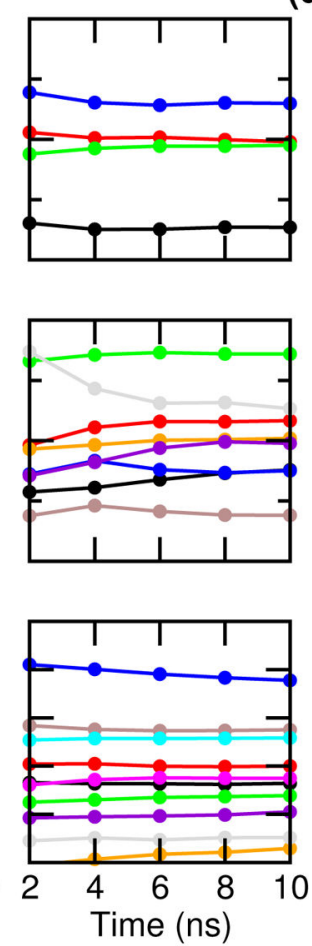

(d)

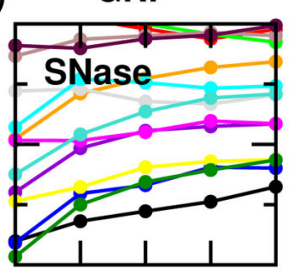

HP36 BBL

- D44 - D129

- E45 - E141

- D46 - H142

- E72

- D145

- E161

- D162

E164

- H166
HEWL SNase

- E7 - E10

- H15 D19

- D18 D21

- E35 - D40

- D48 D43

- D52 - E52

- D66 E57

- D87 - E67

-D101 E73

- D119 E75

D95

- E101

- E122

- E129

- E135

Figure 2.

Convergence of the calculated $\mathrm{p} K_{\mathrm{a}}$ 's for HP36 (a), BBL (b), HEWL (c) and SNase (d). p $K_{\mathrm{a}}$ calculation was performed every $2 \mathrm{~ns}$ per replica based on the cumulative values of the unprotonated fractions at all $\mathrm{pH}$. The left and right panels present the GRF-25 and PMEbased CpHMD simulations. 

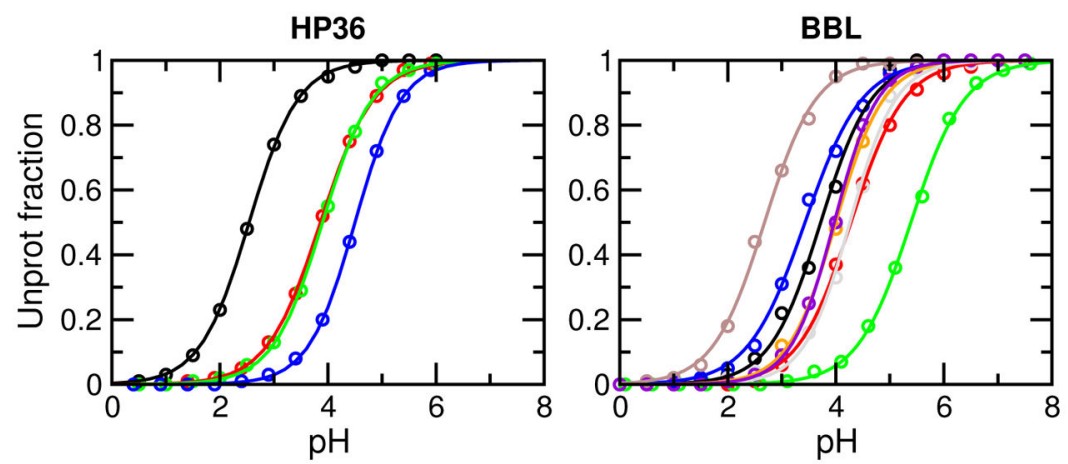

HP36 BBL

- D44 O D129

- E45 ○ E141

- D46 $\circ$ H142

- E72 ○ D145

- E161

- D162

- E164

○ $\mathrm{H} 166$
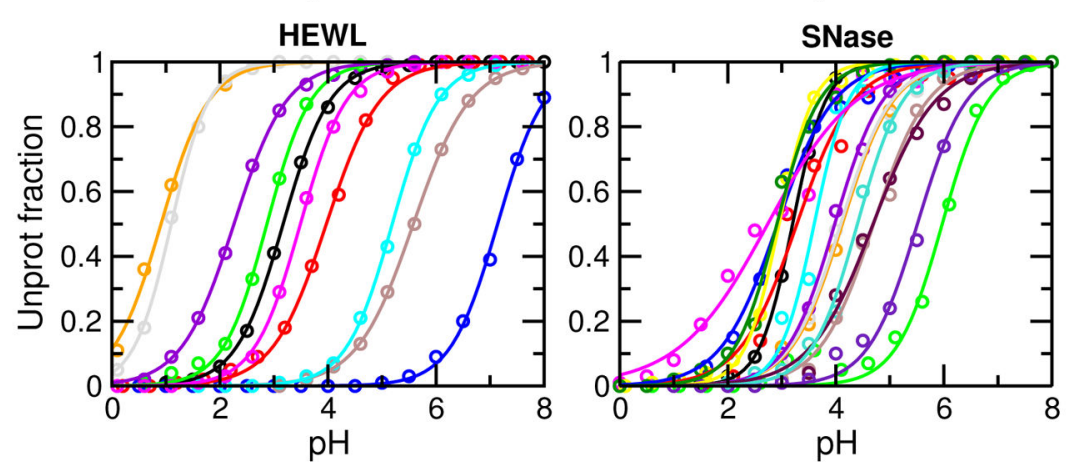

HEWL SNase

- E7 O E10

- H15 O D19

- D18 ○ D21

- E35 ○ D40

- D48 E43

- D52 - E52

D66 E57

- D87 ○ E67

- D101 E73

- D119 ○ E75

D95

- E122

- E129

Figure 3.

Titration plots of HP36, BBL, HEWL and SNase. Unprotonation fractions at different $\mathrm{pH}$ were calculated based on the 10-ns CpHMD simulations. Solid curves represent best fits to the generalized HH equation. For all curves, chi-square of fitting is in the range 0.000170.038 and correlation coefficient is in the range $0.9955-0.999965$. 
(a)
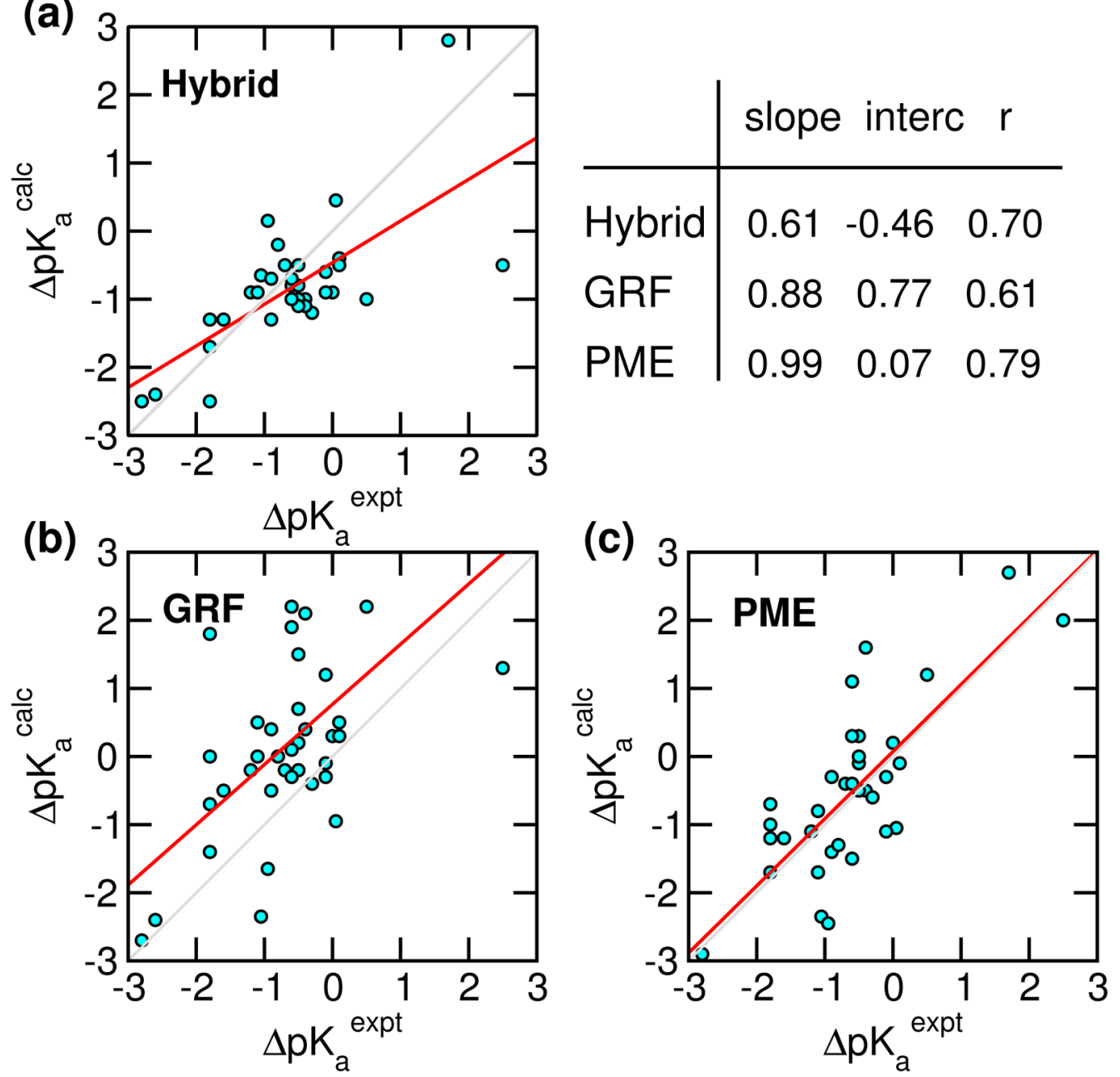

Figure 4.

Correlation between the experimental and calculated $\mathrm{p} K_{\mathrm{a}}$ shifts relative to model compound values for hybrid-solvent, GRF-, and PME-based CpHMD simulations. The diagonal gray line is added to guide the eye. The red line represents linear regression with the slope, intercept (interc) and correlation coefficient ( $\mathrm{r}$ ) indicated. The corresponding $\mathrm{p} K_{\mathrm{a}}$ 's are given in Table 2. 


\section{Table 1}

Calculated $\mathrm{p} K_{\mathrm{a}}$ 's for model Asp, Glu and His

\begin{tabular}{lllll}
\hline Model & Ref & Hybrid & GRF & PME \\
\hline Asp & 4.0 & $3.8 \pm 0.02$ & $3.5 \pm 0.17$ & $4.0 \pm 0.09$ \\
Glu & 4.4 & $4.1 \pm 0.01$ & $4.1 \pm 0.13$ & $4.5 \pm 0.09$ \\
His & 6.5 & $6.9 \pm 0.01$ & $6.8 \pm 0.10$ & $6.5 \pm 0.02$ \\
His-N & 6.6 & - & - & $6.7 \pm 0.03$ \\
His-N & 7.0 & - & - & $6.9 \pm 0.03$ \\
Lys & 10.4 & $10.4 \pm 0.02$ & $10.4 \pm 0.12$ & $10.4 \pm 0.04$ \\
\hline
\end{tabular}

Average $\mathrm{p} K_{\mathrm{a}}$ 's and standard deviations based on five independent sets of $\mathrm{pH}$-replica CpHMD simulations. Reference $\mathrm{p} K_{\mathrm{a}}$ 's refer to the experimentally measured $\mathrm{p} K_{\mathrm{a}}$ 's of blocked single amino acids. ${ }^{51}$ The microscopic $\mathrm{p} K_{\mathrm{a}}$ 's of His-N $\delta_{\text {and His-N }}{ }^{\mathcal{E}}$ are taken from Ref. ${ }^{52}$ All these values are identical to the previous CpHMD work. ${ }^{6-8,25}$ The hybrid-solvent simulation used 5 replicas, each of which was sampled for 10 ns with $\lambda$-update every $10 \mathrm{MD}$ steps. The GRF-based simulation used $10 \mathrm{pH}$ replicas, each of which was sampled for $10 \mathrm{~ns} .25 \mathrm{The}$ PME-based simulation used $5 \mathrm{pH}$ replicas, each of which was sampled for $5 \mathrm{~ns}$. The PMF functions with parameters slightly adjusted to minimize the deviations from the reference $\mathrm{p} K_{\mathrm{a}}$ 's were used (see Table S5). 


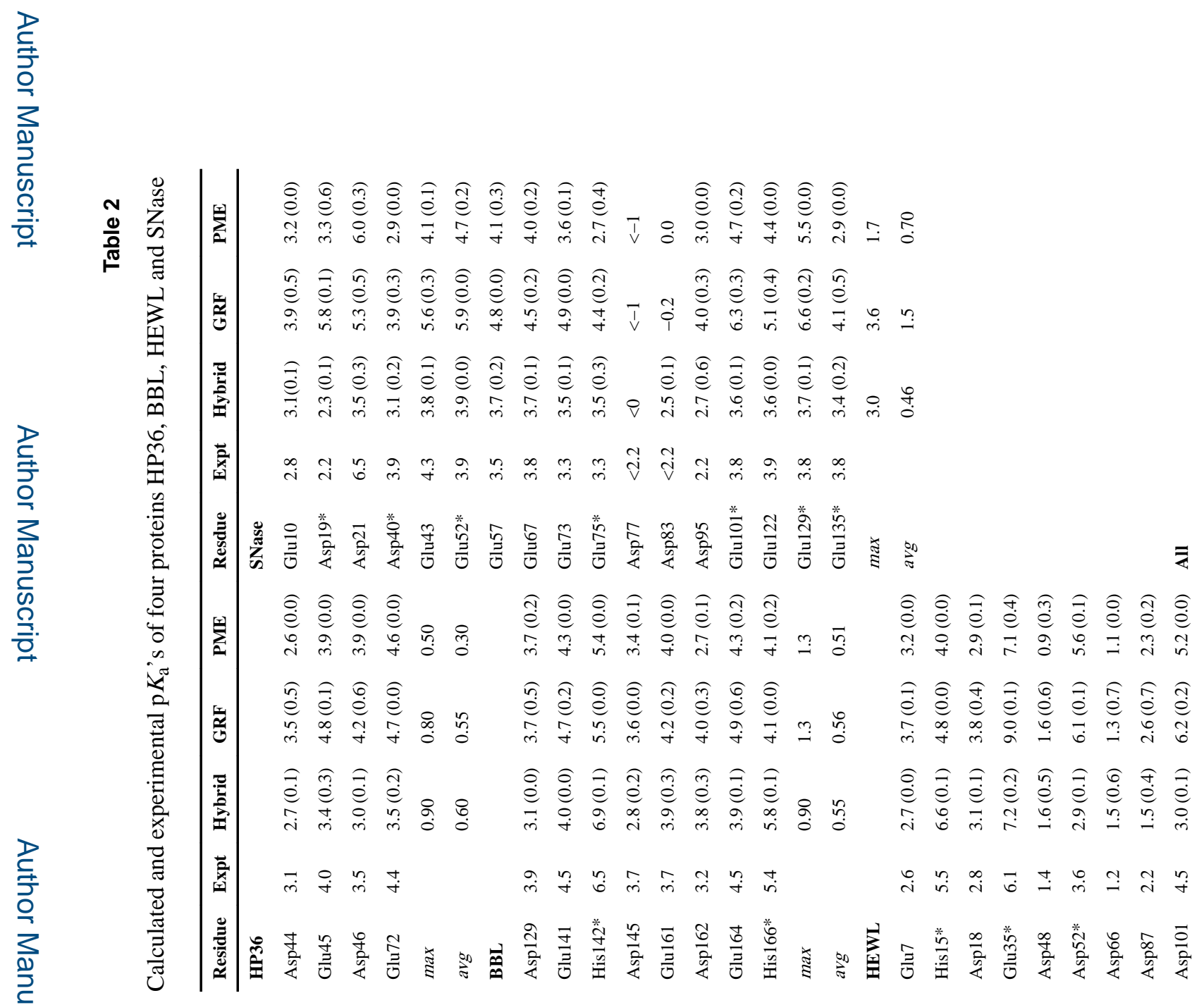




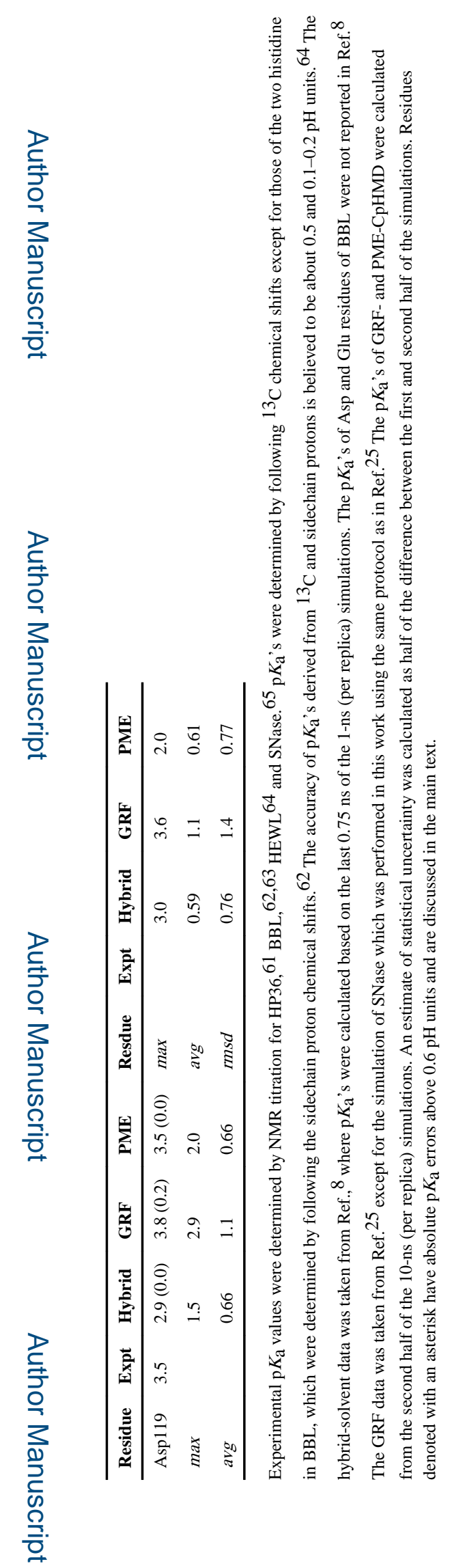

J Chem Theory Comput. Author manuscript; available in PMC 2017 December 04. 